\title{
The Call for Global Health Research Mentorship for Doctorally Prepared Nurses in Low- and Middle-Income Countries
}

\author{
Julie M. Buser, ${ }^{1 \star}$ John N. Cranmer, ${ }^{2,3}$ Veronica M. Dzomeku, ${ }^{4}$ Alice Ngoma-Hazemba, ${ }^{5}$ Tom Ngabirano, ${ }^{6}$ Ashura Bakari, ${ }^{7}$ and \\ Jody R. Lori ${ }^{8}$ \\ ${ }^{1}$ Fogarty International Center, National Institutes of Health, Global REACH, University of Michigan Medical School, Ann Arbor, Michigan; ${ }^{2}$ Emory \\ Ethiopia Implementation Research on Obstetric Mortality, Addis Ababa, Ethiopia; ${ }^{3}$ Emory University, Atlanta, Georgia; ${ }^{4}$ Department of Nursing, \\ College of Health Sciences, Kwame Nkrumah University of Science and Technology, Kumasi, Ghana; ${ }^{5}$ Department of Community and Family \\ Medicine, School of Public Health, University of Zambia, Lusaka, Zambia; ${ }^{6}$ Department of Nursing, College of Health Sciences, Makerere \\ University, Kampala, Uganda; ${ }^{7}$ Ghana Health Service, Suntreso Government Hospital, Kumasi, Ghana; ${ }^{8}$ Department of Health Behavior and \\ Biological Sciences, PAHO/WHO Collaborating Center, School of Nursing, University of Michigan, Ann Arbor, Michigan
}

\begin{abstract}
Limited research about nursing mentorship in low- and middle-income countries (LMICs) is holding science back. This article describes the strengths and challenges associated with global health research mentorship for doctorally prepared nurses whose scholarship focuses on LMICs. Using reflexive narrative accounts from current and former nurse mentors and nurse mentees who participated in a $\mathrm{NIH}$-funded global health doctoral research program, emerging themes revealed the perspectives of mentors and mentees, producing a global health mentoring model for nursing research mentorship relevant to LMICs. Identified themes, which applied across roles and primary affiliations, included 1) collaborative mentor-mentee relationships and 2) enthusiasm for global health nursing. Our global health nursing research mentor-mentee interaction systems conceptual model focuses on nursing science mentoring in LMICs incorporating interpersonal, institutional, and cultural factors. We describe successful components of global nurse researcher mentorship and summarize directions for future research in the field. Our model can be used to create more effective menteecentered mentoring for nurses or health professionals conducting global research. To advance science, we encourage doctorally prepared nurses to support mentee-centered research mentorship experiences that are sensitive to the unique needs of interdisciplinary global health scholarship.
\end{abstract}

\section{INTRODUCTION}

Even though nurses provide most clinical care globally and are the largest occupational group in the health sector, they often receive minimal support and resources to improve global health nursing practice. ${ }^{1}$ Nursing research informs professional practice with sound nursing science contributing to solutions that directly impact patient care and provide global health solutions. ${ }^{2,3}$ Therefore, robust research mentorship for doctorally prepared nurses in low- and middleincome country (LMIC) settings is essential to advance global health nursing science.

Limited research about nursing mentorship in LMICs is holding science back. Cultivating strong mentor-mentee relationship is critical to the success and sustainability of mentorship programs. ${ }^{4,5}$ Mentorship is a dynamic reciprocal interaction between the mentor and mentee that should be beneficial to both members of the dyad. ${ }^{4}$ Mentors can learn and grow professionally from this "two-way street" of learning that occurs between mentors and mentees. Solid mentorship may strategically promote mutual learning and codevelopment of mentor-mentee skills across institutional and income hierarchies common to global health. ${ }^{6}$ The most effective and transformational mentors encourage mentees to question the status quo, propose innovative approaches, and engage in scientific debates with more senior scientists. Despite the value of questioning the status quo in scientific discovery, similar inquiry into academic and institutional cultures is not valued across hierarchal institutions-including some LMIC academic centers. ${ }^{7}$

*Address correspondence to Julie M. Buser, Fogarty International Center, National Institutes of Health, Global REACH, University of Michigan Medical School, Victor Vaughan Bldg., 2nd Floor, 1111 East Catherine St., Ann Arbor, MI 48109. E-mail: jbuser@umich.edu
This article highlights the unique mentorship needs for nurses conducting research in LMICs as a response to the previously published supplement published by the American Journal of Tropical Medicine and Hygiene. ${ }^{8}$ The supplement acted as a catalyst for LMIC research organizations to institutionalize mentorship training and provided a useful guide for the development of robust mentorship training programs at LMIC institutions. ${ }^{5}$ Although global health research on mentorship is well documented in the supplement, there is a gap in the literature focusing specifically on doctorally prepared nurses' mentoring relationships.

\section{METHODS}

The purpose of this article, based on actual lived experience, was to describe current strengths and challenges of global health research mentorship for doctorally prepared nurses whose scholarship focuses on LMICs. We also illustrate how elements of successful global health research mentorship for nurses in LMIC settings vary depending on whether the mentee's primary affiliation for the research is based in a high-income country (HIC) or an LMIC. Authors collected reflexive narrative accounts via email from all known current and former mentors and nurse mentees in an $\mathrm{NIH}$-funded global health research program. Authors analyzed the narratives from seven mentors and mentees based on their role (mentor versus mentee) and primary affiliation (HIC versus LMIC). Next, authors used the reflections to identify themes incorporating four phases of theme development: "initialization," "construction," "rectification," and "finalization"9 and modify an established mentorship framework of LMIC mentorship by Prasad and others. ${ }^{4}$ Based on this process, we identified novel aspects of nursing mentorship for LMIC research. These findings were synthesized 
into the modified conceptual framework sensitive to LMIC nursing science.

Mentees and mentors contributing to this article participated in programs sponsored by the NIH Fogarty International Center in partnership with several NIH institutes and offices, including the Fogarty Global Health Fellows and Research Career Development programs. The Global Health Program for Fellows and Scholars is a 12-month clinical research training program for postdoctorate mentees and doctoral students in the health professions, supports U.S. university consortia to provide collaborative, and mentored global health research training opportunities in LMICs. ${ }^{10,11}$ Health professional mentees are paired with two mentors-one from an HIC institution and another from an LMIC where the research is conducted.

Another global health research program open to nurses and other health professionals involves the Fogarty Emerging Global Leader Award to provide research support and protected time to a research scientist from an LMIC with a junior faculty position at an LMIC academic or research institution leading to an independently funded research career. ${ }^{12}$ Many of the past Fogarty Fellows credit their success to the quality of mentorship they received during the program. ${ }^{7}$

\section{RESULTS}

Themes identified in mentor-mentee observations were applicable across roles and primary affiliations and included 1) collaborative mentor-mentee relationships and 2) enthusiasm for global health nursing. Illustrating the theme of collaborative mentor-mentee relationships, a mentor offered the following observations:

I view the mentor-mentee relationship as a collaborative effort. We are first and foremost colleagues. I strived to role-model a form of participatory research that enhances the role of nursing research in areas of the world where nursing and research are often undervalued. My role is to support the acquisition of research skills, gaining an understanding of cultural, social, and environmental differences, understanding the reciprocal nature of conducting research outside one's own country, and opening doors for networking and future collaborations. All mentees bring their own lived experiences to the research. Many have had years of clinical experience that has shaped their thinking and led them to their current research path. They all have an enormous amount of energy and enthusiasm for the research process. It helps me see things through a new and fresh lens.

Exemplifying the enthusiasm for global health nursing theme, a mentee reflected:

Enthusiasm and commitment to global health research were key characteristics of my mentors. My mentor was critical in helping me acclimate to a different culture and research environment. I embraced my mentor-mentee relationships as reciprocal and strived to accept criticism graciously. While conducting research, I appreciated the openness of communication with members of my mentorship team. I value a mentor who encourages independent behavior yet is approachable and available. In a good mentor, I look for a generosity of spirit and willingness to help me succeed professionally. I seek an expert guide who is keen to share their knowledge of global health freely and serve as a positive role model.

Synthesized perspectives: mutually beneficial nursing research mentorship in LMICs. Based on the formative evaluation of narratives from nurse mentoring relationships in NIH-funded research fellowships, we adapted Prasad and others' systems of interaction between the mentor and mentee to create a Global Health Nursing Research Mentor-Mentee Interaction Systems Conceptual Model (Figure 1). Our model focuses on nursing science mentoring in LMICs incorporating interpersonal, institutional, and cultural factors.

Characteristics of positive mentor-mentee relationships. Mutually beneficial mentee-mentor relationships built during global health nursing research programs included good communication, active listening, and an exchange of honest feedback. Reciprocity and recognition of differences in research environments between HICs and LMICs were valued in positive mentee-mentor relationships. In addition, increased research productivity was expressed as one key output of successful mentoring.

Having a shared interest in research topics and methodology was also crucial for effective mentoring. For HIC mentees, prior exposure to LMIC settings was deemed important in developing a solid foundation for successful relationships. Preexisting research skills for mentees also helped foster productive relationships sparked in large part by aligning skills, methods, and interests. Mentors and mentees from HICs and LMICs valued the characteristics that emerged in their respective relationships. Figure 2 outlines the mentor and mentee characteristics appreciated by participants from both HICs and LMICs.

Contextual factors. Several contextual factors influence global health nursing research in LMICs. The research environment in LMICs varies widely, and the concept of mentorship is frequently not formalized. Often, senior nursing faculties in LMICs have limited experience mentoring nurse researchers. These senior nurses potentially received little-tono mentoring and may not have the skills to mentor early career researchers. Nurses conducting global health research in LMICs are more likely than those in HICs to find themselves in complicated situations where they need to navigate longstanding contextual factors for which they have limited skill or understanding as outsiders (e.g., gender, shifting power, or cultural dynamics). Flexibility is required by nurse mentees from HICs working in LMICs to cope with cultural differences and unanticipated situations that might delay data collection. Host-country funding to support research in LMICs is rare, and the impact of funding from the HIC home institution affects the length of time a nurse can spend conducting research.

\section{DISCUSSION}

We described current strengths and challenges of global health research mentorship for doctorally prepared nurses whose scholarship focuses on LMICs. We also illustrated how elements of successful global health research mentorship for nurses in LMIC settings vary depending on whether mentee's 
Systems of Interaction between the Mentor and Mentee
Mentor-Mentee Relationship Factors

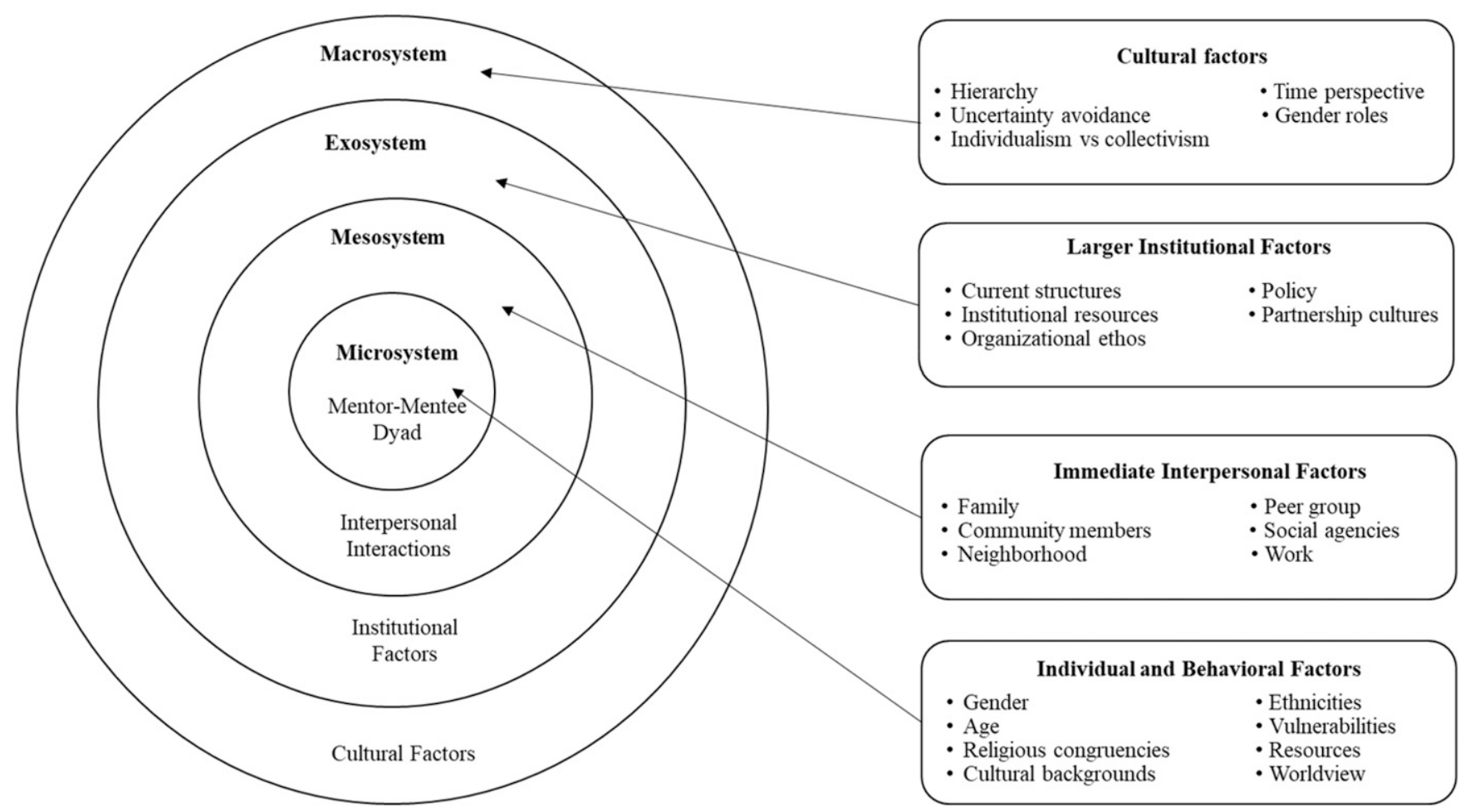

FigURE 1. Global health nursing research mentor-mentee interaction systems (adapted from Prasad et al.). ${ }^{4}$ primary affiliation for the research is based in a HIC or an LMIC. The adapted conceptual model for mentorship can be used to create effective mentee-centered mentoring in global health nursing and other health care disciplines in LMICs. Our Global Health Nursing Research Mentor-Mentee Interaction Systems Conceptual Model focuses on the addition of nursing science in LMICs incorporating interpersonal, institutional, and cultural factors. We described successful components of global nurse researcher mentorship.

Global health nursing research programs that nurture collaborative mentoring teams from HICs and LMICs offer powerful tools to foster sustainable career growth and progress of doctoral prepared nurses conducting their scholarship in LMICs. In general, mentors for nurses conducting global health research should have enough experience with the clinical and/or geographical settings within which the mentee will be operating to ensure productivity and a fruitful learning experience. Global health nursing research programs that promote the need for and benefits of peer mentoring aimed at nurses conducting research in LMICs would be useful. In addition, the importance of establishing a formal, sustainable mentorship culture at LMIC institutions to develop competencies and increase the potential pool of mentors should be emphasized.

Successful global health research and practice depends on strong mentorship, which requires training, guidelines, and standards for mentees, mentors, and mentoring institutions. ${ }^{13}$ To improve nursing science, we encourage doctorally prepared nurse scientists to advocate for successful and satisfying research mentorship, and participate in collaborative interdisciplinary global health fellowship programs such as the $\mathrm{NIH}$ Fogarty Fellows and Research Career Development programs.

Limitations. This research was limited by a small sample size that included those mentors and mentees participating in Fogarty International Center programs and did not explore the

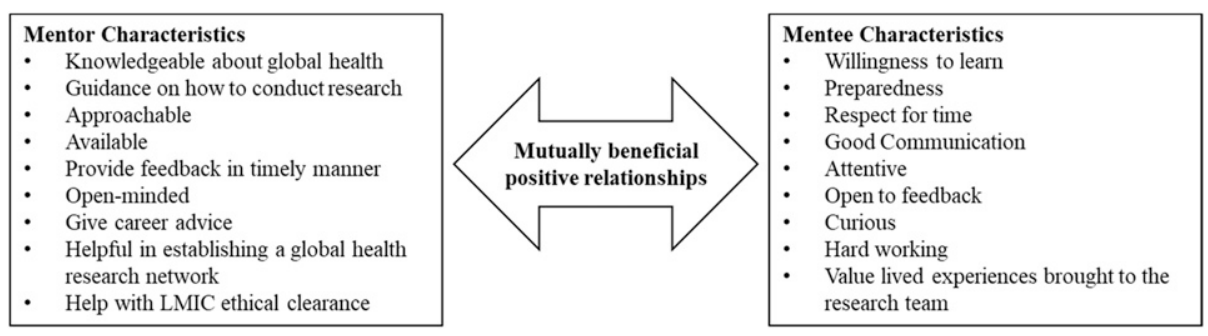

FIGURE 2. Mentor and mentee characteristics of mutually beneficial nursing research mentorship in low-and middle-income countries (LMICs). 
mentorship perspective for those engaged in other global health nursing research programs in LMIC settings. Despite the sample size, this research provides a new perspective from which to review global health research mentorship for doctorally prepared nurses conducting research in multiple LMICs.

\section{CONCLUSION}

The Global Health Nursing Research Mentor-Mentee Interaction Systems Conceptual Model can be used to create more effective mentee-centered mentoring for nurses conducting global health research. Without effective global health research mentorship, nursing science will continue to lag behind. Increased emphasis on developing strong global health research mentorship programs for doctorally prepared nurses in LMIC settings is necessary as we strive to improve scientific discovery and context-specific solutions, and reduce global health disparities between and within countries. Moving forward, we advocate for additional interdisciplinary collaboration and leadership support to build capacity in global health nursing research mentorship.

Received December 18, 2020. Accepted for publication February 5, 2021.

Published online March 22, 2021.

Authors' addresses: Julie M. Buser, Fogarty International Center, National Institutes of Health, Global REACH, University of Michigan Medical School, Ann Arbor, MI, E-mail: jbuser@umich.edu. John N. Cranmer, Emory Ethiopia Implementation Research on Obstetric Mortality, Addis Ababa, Ethiopia, and Emory University, Atlanta, GA, E-mail: john.cranmer@emory.edu. Veronica M. Dzomeku, Department of Nursing, College of Health Sciences, Kwame Nkrumah University of Science and Technology, Kumasi, Ghana, E-mail: vmdzomeku@ gmail.com. Alice Ngoma-Hazemba, Department of Community and Family Medicine, School of Public Health, University of Zambia, Lusaka, Zambia, E-mails: alicengomah@gmail.com or ahazemba@ unza.zm. Tom Ngabirano, Department of Nursing, College of Health Sciences, Makerere University, Kampala, Uganda, E-mail: tomngabirano@gmail.com. Ashura Bakari, Ghana Health Service, Suntreso Government Hospital, Kumasi, Ghana, E-mail: abakari@ yahoo.com. Jody R. Lori Department of Health Behavior and Biological Sciences, PAHO/WHO Collaborating Center, School of Nursing, University of Michigan, Ann Arbor, MI, E-mail: jrlori@ umich.edu.

\section{REFERENCES}

1. World Health Organization, 2020. State of the World's Nursing 2020: Investing in Education, Jobs and Leadership. Accessed December 9, 2020.

2. Scala E, Price C, Day J, 2016. An integrative review of engaging clinical nurses in nursing research. J Nurs Scholarsh 48: 423-430.

3. Baltzell K, McLemore M, Shattell M, Rankin S, 2017. Impacts on global health from nursing research. Am J Trop Med Hyg 96: 765-766.

4. Prasad S, Sopdie E, Meya D, Kalbarczyk A, Garcia PJ, 2019. Conceptual framework of mentoring in low-and middle-income countries to advance global health. Am J Trop Med Hyg 100 (Suppl 1): 9-14.

5. Chi BH, Belizan JM, Blas MM, Chuang A, Wilson MD, Chibwesha CJ, Farquhar C, Cohen CR, Raj T, 2019. Evaluating academic mentorship programs in low-and middle-income country institutions: proposed framework and metrics. Am J Trop Med Hyg 100 (Suppl 1): 36-41.

6. Cole DC, Johnson N, Mejia R, McCullough H, Turcotte-Tremblay AM, Barnoya J, Falabella Luco S, 2016. Mentoring health researchers globally: diverse experiences, programmes, challenges and responses. Glob Public Health 11: 1093-1108.

7. Katz F, Glass RI, 2019. Mentorship training is essential to advancing global health research. Am J Trop Med Hyg 100 (Suppl 1): 1-2.

8. Am J Trop Med Hyg, 2019. Mentoring in low-and middle-income countries to advance global health. Am J Trop Med Hyg 100 (Suppl 1): 1-53.

9. Vaismoradi M, Jones J, Turunen H, Snelgrove S, 2016. Theme development in qualitative content analysis and thematic analysis. J Nurs Educ 6: 100-110.

10. $\mathrm{NIH}, 2020$. Global Health Program for Fellows and Scholars. Available at: https://www.fic.nih.gov/Programs/Pages/scholarsfellows-global-health.aspx. Accessed December 9, 2020.

11. University of Washington, 2020. Fogarty Fellows Overview. Available at: http://fogartyfellows.org/. Accessed December 9, 2020.

12. NIH, 2017. Research Career Development Awards. Available at: https://researchtraining.nih.gov/programs/careerdevelopment/ K43\#: :text=The\%20purpose\%20of\%20the\%20Fogarty,an\% 20 independently \%20funded\%20research\%20career. Accessed December 9, 2020.

13. Hansoti B, Kalbarczyk A, Hosseinipour MC, Prabhakaran D, Tucker JD, Nachega J, Wallis L, Stiles JK, Wynn A, Morroni C, 2019. Global health mentoring toolkits: a scoping review relevant for low-and middle-income country institutions. Am J Trop Med Hyg 100 (Suppl 1): 48-53. 